\title{
Different Methods analysing Convection-Diffusion
}

\author{
Stefanie Winkler ${ }^{1 *}$, Martin Bicher ${ }^{1,2}$ \\ ${ }^{1}$ Institute for Analysis and Scientific Computing, Vienna University of Technology, Wiedner Haupstraße 8-10, \\ 1040 Vienna, Austria; *stefanie.winkler@tuwien.ac.at \\ ${ }^{2}$ dwh GmbH, Neustiftgasse 95-97, 1070 Vienna, Austria
}

Simulation Notes Europe SNE 25(1), 2015, 43 - 48

DOI: $10.11128 /$ sne.25.tn. 10281

Received: November 8, 2014; Revised January 9, 2015;

Accepted: February 15, 2015;

Abstract. Many countries in this world have lack of drinking water. Austria has advantage of drinking water coming from the mountains. This article contains a study focusing on mathematical modelling using different methods for the analysis of groundwater pollution. The distribution of pollution follows the convection-diffusion equation. Therefore different methods ranging from analytical and numerical to alternative approaches dealing with random walk are compared. The analysis of the approaches is mostly done for one and two dimensional case.

\section{I ntroduction}

In order to analysis the po llution distribution in water of sim ilar circum stances the $m$ athematical equation describing $\mathrm{t}$ his be haviour i $\mathrm{s}$ a $\mathrm{c}$ onvection-diffusion equation. This equation can not only be used to analysis the be haviour of pollution. Also in biology, chem istry and ot her fiel ds of study $t$ his e quation i s im portant. Regarding biology the equation can be use $d$ to predict the development of fur pattern for cats. In chemistry the mixture of different substances follows this equation. In the field of physical modelling and simulation this equation is often $\mathrm{c}$ alled heat equation bec ause it describe $\mathrm{s}$ the distribution of heat em anating from a source. De spite disci plines in nat ural sciences also the fi nance market uses this equation $t$ o foresee the behaviour of buyers of st ocks. In general the convection- diffusion equation looks as follows:

$$
\frac{\partial \mathrm{c}}{\partial \mathrm{t}}=\mathrm{D} \cdot \nabla^{2} c-v \cdot \nabla c
$$

Equation (1) is a partial differential equation of second or der a nd c ontains tw o dif ferent variables $\mathrm{D}, \mathrm{v}$ which can be ti me-dependent, position-dependent or simply constant. In the following we assume that all the variables are constant. The first term of this equation describes a re gular distribution in every direction. It is similar to spreading of waves after throwi ng in a little stone into water. The variable $\mathrm{v}$ in the second term of (1) symbolises the velocity field of oriente $d$ movement. Assuming for example a river with a ce rtain flux the $n$ the distribution would be influence by the velocity of the flux. This information will be transformed into $t$ he equation using the variable $\mathrm{v}$. To sum it up, the convection-diffusion equation contains one part de scribing the chaotic movement in all dir ections and an orie nted distribution depending on the circumstances. In the following a flux only in $\mathrm{x}$-direction is assumed. This problem description wi ll be a nalysed usi ng three different a pproaches applied in one and two dimensions.

\section{Analytical Solution}

In this case, due to the used initial and boundary conditions, an anal ytical solutio $\mathrm{n}$ can be given. The initial condition describes a pollutio $n$ sources which releases all the pollution at ti me $t=0$ without inj ecting any further pollution. B oth so lutions, one- and twodimensional, are used to validate the different methods. One-dimensional. Using the regarded equation is given as follows

$$
\frac{\partial \mathrm{c}}{\partial \mathrm{t}}=\mathrm{D} \cdot \frac{\partial^{2} \mathrm{c}}{\partial \mathrm{x}^{2}}-v \cdot \frac{\partial \mathrm{c}}{\partial \mathrm{x}}
$$

and has to fulfill the initial $c\left(x_{0}, 0\right)=\delta(x)$ and the boundary conditions $\lim _{x \rightarrow \pm \infty} c(x, t)=0$. Using substitutions described in [1] the equation (2) can be written as

$$
\begin{array}{cc}
\tau=D t, & b=\frac{v}{D} \\
\mathrm{y}=\mathrm{x}-\mathrm{b} \tau, & \mathrm{y}_{0}=\mathrm{b} \tau_{0} \\
\frac{\partial \mathrm{c}(\mathrm{y}, \tau)}{\partial \tau}=\frac{\partial^{2} \mathrm{c}(\mathrm{y}, \tau)}{\partial \mathrm{y}^{2}}
\end{array}
$$


The resulting line in equation (3) can be multiplied by $e^{-p \tau}$. After integration with respect to $\tau$ one obtains an ordinary differential equation which can be solved using basic $m$ athematical tools. A La place ba ck tra nsformation and backward substitution gives the solution of equation (2).

$$
c(x, t)=\frac{1}{\sqrt{4 \pi D t}} e^{-\frac{(x-v t)^{2}}{4 D t}}
$$

Two-dimensional. In the tw o dimensional case the obtained equation changes to

$$
\frac{\partial \mathrm{c}}{\partial \mathrm{t}}=\mathrm{D} \cdot \frac{\partial^{2} \mathrm{c}}{\partial \mathrm{x}^{2}}+\mathrm{D} \cdot \frac{\partial^{2} \mathrm{c}}{\partial \mathrm{y}^{2}}-v \cdot \frac{\partial \mathrm{c}}{\partial \mathrm{x}}
$$

Analogue to the one-dimensional case certain initial and boundary conditions are defined as follows

$$
\begin{gathered}
\mathrm{c}\left(\mathrm{x}_{0}, \mathrm{y}_{0}, 0\right)=\delta(x) \delta(y) \\
\lim _{x, y \rightarrow \infty} c(x, y, 0)=0 \\
\lim _{x, y \rightarrow-\infty} c(x, y, 0)=0
\end{gathered}
$$

In order to $s$ olve equation (5) a s pecific form of the solution is assumed.

$$
c(x, y, t)=g_{1}\left(x, x_{0}, t\right) g_{2}\left(y, y_{0}, t\right)
$$

The $\mathrm{f}$ unctions $g_{1}$ and $g_{2}$ are so lutions of $\mathrm{t}$ he on edimensional convection- diffusion equation with $\mathrm{c}$ onstant coe fficients. Therefore $g_{1}$ and $g_{2}$ can be taken from the one-dimensional analytical solution (4).

$$
\begin{aligned}
\mathrm{g}_{1}\left(\mathrm{x}, \mathrm{x}_{0}, \mathrm{t}\right) & =\frac{\mathrm{A}_{1}}{2 \sqrt{D \pi t}} e^{-\frac{\left(x-x_{0}-v t\right)^{2}}{4 D t}} \\
\mathrm{~g}_{2}\left(\mathrm{y}, \mathrm{y}_{0}, \mathrm{t}\right) & =\frac{\mathrm{A}_{2}}{2 \sqrt{D \pi t}} e^{-\frac{\left(y-y_{0}\right)^{2}}{4 D t}}
\end{aligned}
$$

The source is located at the origin the refore the val ues $x_{0}=0$ and $y_{0}=0$ can $\mathrm{b}$ e in serted. Additionally the integral over the whole domain has to be 1 .

$$
\begin{gathered}
1=\int_{-\infty}^{\infty} \int_{-\infty}^{\infty} c(x, y, t)= \\
=\int_{-\infty}^{\infty} g_{1}(x, 0, t) d x \int_{-\infty}^{\infty} g_{2}(y, 0, t) d y=A_{1} A_{2}
\end{gathered}
$$

This integration result leads to the analytical solution in two dimensions.

$$
c(x, y, t)=\frac{1}{4 D t \pi} e^{-\frac{(x-v t)^{2}}{4 D t}}
$$

\section{Numerical Approximation}

This section introduces two t ypes of numerical approximations. On the one hand there is the finite diffe rence method (FDM). In this approximation the derivative of the $\mathrm{d}$ ifferential eq uation is approached by tak ing the difference quotient of the neighboring grid points. The method is easy to use but slightly weak concerning the accuracy. $\mathrm{T}$ he second $\mathrm{m}$ ethod is the finite elem ent method (FEM) and is based on formulating variations of the differential equation. FEM determines approximated solutions c onsisting of piece wise de fined polynomials on a fi ne resolution of the domain. The a dvantage of FEM is the suitability for any geometry.

\subsection{Finite Difference Method}

One-dimensional. Using finite diffe rences to approximate the first and seco nd derivatives the partial differential equation (2) tra nsforms into an or dinary differential equation.

$$
\frac{\mathrm{dc}}{\mathrm{dt}}=\mathrm{D} \cdot \frac{\mathrm{c}_{\mathrm{i}+1}-2 \mathrm{c}_{\mathrm{i}}+\mathrm{c}_{\mathrm{i}-1}}{d x^{2}}-v \cdot \frac{c_{i}-c_{i-1}}{d x}
$$

The time derivative can be replaced as follows

$$
\frac{\mathrm{dc}}{\mathrm{dt}}=\frac{\mathrm{c}^{\mathrm{k}+1}-\mathrm{c}^{\mathrm{k}}}{\Delta \mathrm{t}}
$$

Using (11) equation (10) can also be written as a matrix product

$$
\frac{\mathrm{c}^{\mathrm{k}+1}-\mathrm{c}^{\mathrm{k}}}{\Delta t}=S \cdot c^{k}
$$

whereas $\mathrm{c}^{\mathrm{k}}$ is the current concentration of pollution and $c^{\mathrm{k}+1}$ the concentration in the next time step. In order to determine $c^{\mathrm{k}+1}$ using the Explicit Euler equation (12) is rearranged.

$$
\mathrm{c}^{\mathrm{k}+1}=(\mathrm{S} \cdot \Delta \mathrm{t}+\mathrm{I}) \mathrm{c}^{\mathrm{k}}
$$

It is well known that the Explicit Euler can be un stable using the wrong step size relation. Notation (12) can be also used to find the Im plicit Euler form ulation. The current concentration on the $r$ ight hand side in equation (13) is re placed by the c oncentration of the future time step in order to obtain the implicit formulation.

$$
\mathrm{c}^{\mathrm{k}+1}=(\mathrm{I}-\mathrm{S} \cdot \Delta \mathrm{t})^{-1} \mathrm{c}^{\mathrm{k}}
$$

Two-dimensional. Regarding the p roblem form ulation in tw o d imensions $t$ he finite di fference $m$ ethod looks a little bit different. Due to the fact that an equidistant grid, $d x=d y$ is used th e approximation can be given as follows

$$
\begin{gathered}
\frac{\mathrm{dc}}{\mathrm{dt}}=\mathrm{D} \cdot \frac{\mathrm{c}_{\mathrm{x}+1, \mathrm{y}}+\mathrm{c}_{\mathrm{x}-1, \mathrm{y}}-4 \mathrm{c}_{\mathrm{x}, \mathrm{y}}+\mathrm{c}_{\mathrm{x}, \mathrm{y}+1}+\mathrm{c}_{\mathrm{x}, \mathrm{y}-1}}{d x^{2}} \\
-v \cdot \frac{c_{x, y}-c_{x-1, y}}{d x}
\end{gathered}
$$

In contrary to the two-dimensional case the matrix nota- 
tion is not as easy as in one dimension.

$$
\mathrm{c}_{\mathrm{x}, \mathrm{y}}^{\mathrm{k}+1}=\mathrm{c}_{\mathrm{x}, \mathrm{y}}^{\mathrm{k}}+h \frac{d c}{d t}
$$

Therefore only the Explicit Euler method is implemented as shown in (15).

\subsection{Finite Element Method}

The finite elem ent method was only realized for the convection-diffusion equation in one dimension.

$$
\begin{gathered}
\frac{\partial \mathrm{c}}{\partial \mathrm{t}}-\mathrm{D} \frac{\partial^{2} \mathrm{c}}{\partial \mathrm{t}^{2}}+\mathrm{v} \frac{\partial \mathrm{c}}{\partial \mathrm{x}}=0 \text { in } \Omega \\
\mathrm{c}=0 \text { on } \partial \Omega
\end{gathered}
$$

First of all the weak solution of (16) is formalized using a test function of the according Sobolev space $\phi \epsilon H_{0}^{1}$.

$$
\int_{\Omega} \frac{\partial \mathrm{c}}{\partial \mathrm{t}} \phi \mathrm{d} \Omega+\int_{\Omega}(\mathrm{D} \nabla \mathrm{c} \nabla \phi+\mathrm{v} \nabla \mathrm{c} \phi)=0
$$

The formulation of the Gale rkin approximation is necessary to form ulate the sol ution e quation of the finite element method.

$$
\mathrm{c}^{\mathrm{n}}(\mathrm{x})=\sum_{\mathrm{j}=1}^{\mathrm{n}} \mathrm{c}_{\mathrm{j}} \varphi_{\mathrm{j}}(\mathrm{x})+\mathrm{c}_{0}(\mathrm{x})
$$

The unk nown variables $c_{j}$ in equation (18) have to be determined. Using linear ba sis functions called 'hatfunctions' for $\varphi$ a linear system of $n$ equations wit $\mathrm{h} n$ unknowns, called the Galerkin formulation, results [3].

$$
\begin{aligned}
& \sum_{j=1}^{n_{e}} \frac{\partial c_{j}}{\partial t} \int_{\Omega} \varphi_{k} \varphi_{i} d \Omega+ \\
& \sum_{\mathrm{j}=1}^{\mathrm{n}_{\mathrm{e}}} \mathrm{c}_{\mathrm{j}} \int_{\Omega}\left(\mathrm{D} \nabla \varphi_{\mathrm{j}} \nabla \varphi_{\mathrm{i}}+\nabla \varphi_{\mathrm{j}} \varphi_{\mathrm{i}}\right) \mathrm{d} \Omega=0
\end{aligned}
$$

In equation (19) $n_{e}$ is the num ber of elem ents in every finite elem ent and $\Omega^{e_{k}}$ is t he domain of ele ment $e_{k}$. Equation (19) can also be written in a short form.

$$
\begin{gathered}
\dot{\mathrm{c}} \cdot M+c \cdot S=0 \\
\mathrm{~m}_{\mathrm{ij}}=\int_{\Omega^{\mathrm{e}_{\mathrm{k}}}} \varphi_{\mathrm{i}} \varphi_{\mathrm{j}} \mathrm{d} \Omega \\
\mathrm{s}_{\mathrm{ij}}=\int_{\Omega^{\mathrm{e}_{\mathrm{k}}}}\left(\mathrm{D} \nabla \varphi_{\mathrm{j}} \nabla \varphi_{\mathrm{i}}+\nabla \varphi_{\mathrm{j}} \varphi_{\mathrm{i}}\right) \mathrm{d} \Omega
\end{gathered}
$$

The matrices of (20) are called mass matrix $M$ and stiffness matrix $S$. Considering the mentioned 'hatfunctions' it is clear, that only a few of the possible integrals are not equal zero.

Those basis functions which corres pond to the corner points of the element will lead to non trivial results. Because the ele ment $i$ is connected to $i-1$ and $i+1$ the profile of the $m$ atrices is a band $m$ atrix with widt $h$ three.

$$
\begin{gathered}
M \frac{\mathrm{c}^{k+1}-c^{k}}{\Delta t}+\theta S c^{k+1}+(1-\theta) S c^{k}=0 \\
0 \leq \theta \leq 1
\end{gathered}
$$

Equation (22) is called $\theta$-method and will be used to present implicit and e xplicit methods for solving (21). The most common values for $\mathrm{q}$ are:

- $\theta=0$, Eplicit Euler

- $\theta=1$, Implicit Euler

- $\theta=\frac{1}{2}$, Implicit Heun

Using this method the Explicit and Im plicit Euler algorithm can be given.

$$
\begin{aligned}
& c^{k+1}=M^{-1}(M-\Delta t S) c^{k} \\
& c^{k+1}=(M+\Delta t S)^{-1} M c^{k}
\end{aligned}
$$

\section{Random Walk}

An alternative method for simulating transport is the socalled random walk. This appro ach is contrary $\mathrm{t} o$ the numerical solutions. $T$ he foc us changes from a macroscopic view $t$ o the sim ulation of $m$ icroscopic be havior of diffusion by analyzing movements of single particles.

\subsection{I ntuitive Approach}

The intuitive approach describes a model which uses no grid or collision rules. It is implemented again for both dimensions.

One-dimensional. At $\mathrm{t}$ he beginning $\mathrm{t}=0$ all the particles are placed in the origin presenting the source of pollution. The pollution injection happens only at $\mathrm{t}=$ 0 . The simulation focuses on the convection and diffusion behaviour of these initial particles. In this approach the movement of particles is described by:

$$
\begin{gathered}
\mathrm{p}_{\text {new }}=\mathrm{p}_{\text {old }} \\
r=X \cdot \Delta x
\end{gathered}
$$

The particle $\mathrm{m}$ otion in (23) consists of three parts. In order to get the new position $p_{\text {new }}$ at time $t+\Delta t$ these three $\mathrm{c}$ omponents a re s ummed up. $\mathrm{T}$ he variable $p_{\text {old }}$ stands for the position at time $t$. The velocity field $v$ is multiplied by the step size. T he variable $r$ describes the diffusive movement of a particle for one time step and is added to the former particle position $p_{\text {old }}$.

The second equation in (23) defines the movement $r$ in particular. It consists of the step size in space $\Delta x$ and a norm ally distribute $\mathrm{d}$ ra ndom variable $X$ with $\mathrm{m}$ ean zero and unit variance. In every time step the new position of ev ery particle is calcu lated with equation (23). 
The sim ulation ends when the chosen simulation tim e tend is reached.

Two-dimensional. For expansion in a tw odimensional domain the movement has to be define $\mathrm{d}$ in a different way. There is no initial velocity but there is an initial direction of every particle $d_{0}$. The diffusive transport is realized by us ing a norm ally distributed random variable $X$ and a uniformly distributed random number $U . X$ is used to generate a random length and $U$ chooses a coincidental direction.

$$
\begin{aligned}
& r=X \cdot \Delta x \quad \alpha=U \cdot 2 \pi \\
& d_{0}=\left(\begin{array}{l}
1 \\
0
\end{array}\right) \quad d_{n+1}=\left(\begin{array}{cc}
\cos \alpha & -\sin \alpha \\
\sin \alpha & \cos \alpha
\end{array}\right) \cdot d_{n}
\end{aligned}
$$

In (24) $r$ stands for the distance the particle moves in a certain tim e s tep. The influence of this param eter is similar to the diffusi on coefficient. $X$ is the mentioned normally distributed random variable and $\Delta x$ describes the step size in space. The s econd equation of (24) sets the direction for the particle's ne xt move. The initial direction $d_{0}$ is only necessary for the recursive de finition. During sim ulation the direction of the last movement is used to calculate the next one. The convection is realized by a shift in $\mathrm{fl}$ ow direction along $x$. The final formulation of the random walk movement can be given as follows

$$
p_{\text {new }}=p_{\text {old }}+d \cdot r+v \Delta t
$$

\subsection{Gaussian Approach}

This approach shows the connection between a random walk approach and the analytical solution.

One-dimensional. The an alytical so lution of th e convection-diffusion e quation (2) is used to de fine the particle movement. Considering the probability density function of a normal or Gaussian distribution

$$
f(x)=\frac{1}{\sqrt{2 \pi \sigma^{2}}} e^{-\frac{(x-\mu)^{2}}{2 \sigma^{2}}}
$$

At the beginning $\mathrm{t}=0$ all the particles are placed in the origin presenting the source of pollution. The pollution injection happens only at $t=0$. The simulation focuses on $t$ he conve ction and diffusion be haviour of $t$ hese initial particles. In this approach the movement of particles is described by:

$$
\begin{gathered}
\mathrm{p}_{\text {new }}=\mathrm{p}_{\text {old }} \\
r=X \cdot \Delta x
\end{gathered}
$$

the formal equivalence to $t$ he analytical solu tion (4) is obvious. The parameters used in (27) stand for the mean value $m$ and the standard deviation $s$ which characterize the position and the width of the Gaussian bell curve in a unique way. Therefore the according parameters in (4) can be read out. [4]

$$
\mu=v \cdot t \quad \sigma^{2}=2 \cdot D t
$$

Due to the properties and meaning of the parameters in (28) the hei ght and wi dth of the conce ntration peak depending on time is given. The corresponding particle movement using (29) can be formulated as follows

$$
p_{\text {new }}=p_{\text {old }}+v \Delta t+\sqrt{2 D \Delta t} \cdot X
$$

The variable $X$ stands for a normally distributed random number with mean zero and unit variance as in the intuitive approach. $X$ is newly generated in eve ry step for each particle. Ide ntifiable by the velocity $v$ the second term stands for $\mathrm{t}$ he convective $\mathrm{m}$ otion. This term is equal to the term of the in tuitive approach. The radical term describes the diffusive motion and is based on the standard derivation.

Two-dimensional. In order to en large this approach in two dim ensions the m ovement along y-direction has to be a dded. For an e xpansion in a tw o-dimensional domain the $y$-component of the $\mathrm{m}$ ovement has $\mathrm{t}$ o be defined. Due to the fact that there is no fl ux the new particle position can be calculated using

$$
\begin{aligned}
& p_{x}^{\text {new }}=p_{x}^{\text {old }}+v \Delta t+\sqrt{2 D \Delta t} \cdot X_{x} \\
& p_{y}^{\text {new }}=p_{y}^{\text {old }}+\sqrt{2 D \Delta t} \cdot X_{y}
\end{aligned}
$$

The variables $X_{x}$ and $X_{y}$ stand for independent normally distributed random numbers which are newly generated in every step for each particle. The term $v \Delta t$ describes the convective transport. Due to the fact that the diffusion coefficient is equal for the $x$ - and $y$-direction the diffusive movement $\sqrt{2 D \Delta t}$ in the random walk definition (30) is the same.

\section{Results}

In the following section the analytical solutions in bot $\mathrm{h}$ dimensions a re com pared $t \mathrm{o}$ the various approac hes. The different conce ntration errors a re discussed. In general the pa rameter settin $\mathrm{g}$ is: diffusion coefficient $D=0.02$ and velocity $v=0.02$.

The step sizes $\Delta t$ and $\Delta x$ are variable. The regarded simulation time varies between $\quad t_{\text {end }}=250$ and $t_{\text {end }}=500$. 


\subsection{Analytical vs. Finite Difference Method Results}

First of all the numerical solutions are considered.

One-dimensional. In the plot below in Figure 1 the red curve i $\mathrm{s}$ the analyti cal solution and the blue line sketches the numerical approximation using the Implicit Euler algorithm.

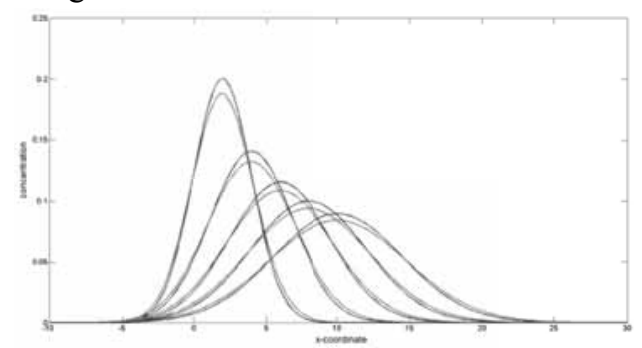

Figure 1. Comparison of the analytical solution and FDMusing matrix notation.

The results in Table 1 show the instability of the Explicit Euler $\mathrm{m}$ ethod. The I mplicit Euler algorithm is not only ultra-stable but also faster and more exact than the Explicit Eu ler. The a pproximation u sing finite differences is well-fitting.

\begin{tabular}{cccccc}
\hline & & \multicolumn{2}{c}{ Explicit } & Euler & \multicolumn{2}{c}{ Implicit } & Euler \\
$\Delta t$ & $\Delta x$ & $\|.\|_{\infty}$ & $\|\cdot\|_{1}$ & $\|.\|_{\infty}$ & $\|\cdot\|_{1}$ \\
\hline 1 & 1 & 0.016 & $4.231 E^{-4}$ & 0.016 & $4.753 E^{-4}$ \\
\hline 1 & $\frac{1}{2}$ & 0.009 & $1.404 E^{-4}$ & 0.010 & $1.600 E^{-4}$ \\
\hline$\frac{1}{2}$ & $\frac{1}{4}$ & 0.005 & $0.831 E^{-5}$ & 0.005 & $7.323 E^{-5}$ \\
\hline$\frac{1}{2}$ & $\frac{1}{16}$ & NaN & NaN & 0.002 & $3.531 E^{-5}$ \\
\hline
\end{tabular}

Table 1. Error values of FEM using Explicit and Implicit Euler.

Two-dimensional. The results re garding t he twodimensional implementation show a similar behaviour. In the following the error values are studied in detail.

Also in the two-dimensional case the Explicit Euler works not for all parameter choices. The error values are again quite good. The finite difference meth od of the two-dimensional dom ain approximates the convec tiondiffusion equation in an appropriate way.

\begin{tabular}{|c|c|c|c|}
\hline \multirow[b]{2}{*}{$\Delta t$} & \multirow[b]{2}{*}{$\Delta x$} & \multicolumn{2}{|c|}{ Explicit Euler } \\
\hline & & $\|\cdot\|_{\infty}$ & $\|\cdot\|_{1}$ \\
\hline 1 & 1 & 0.027 & $1.5624 E^{-4}$ \\
\hline 1 & $\frac{1}{2}$ & 0.017 & $3.779 E^{-5}$ \\
\hline$\frac{1}{2}$ & $\frac{1}{4}$ & $9.148 E^{-4}$ & $1.464 E^{-5}$ \\
\hline
\end{tabular}

\begin{tabular}{cccc}
\hline$\frac{1}{4}$ & $\frac{1}{8}$ &.$E^{119}$ &.$E^{120}$ \\
\hline
\end{tabular}

Table 2. The error values for FDM are shown.

\subsection{Analytical vs. Finite Element Method Results}

The accuracy of the finite element method is better than of the finite difference method.

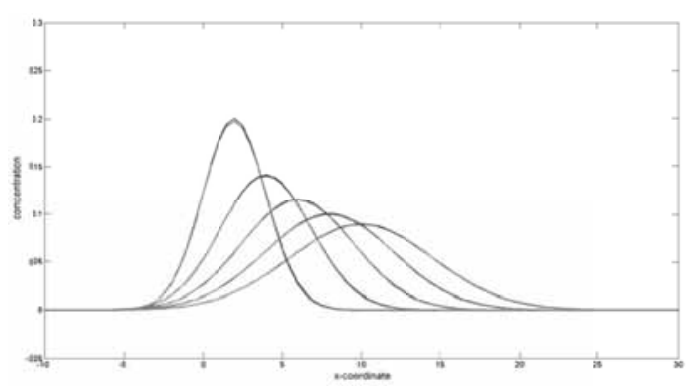

Figure 3. The error for the Implicit Euler algorithm of the FEM is shown.

In Figure 3 above the upper plot shows the analytical solution as well as the finite element method using Implicit Euler. It is hard to distinguish the different curves.

\begin{tabular}{|c|c|c|c|c|c|}
\hline$\Delta t$ & $\Delta x$ & $\begin{array}{l}\text { Explicit } \\
\|\cdot\|_{\infty}\end{array}$ & $\begin{array}{l}\text { Euler } \\
\qquad\|\cdot\|_{1}\end{array}$ & $\begin{array}{l}\text { Implicit } \\
\|.\|_{\infty}\end{array}$ & $\begin{array}{l}\text { Euler } \\
\qquad\|\cdot\|_{1}\end{array}$ \\
\hline 1 & 1 & $7.18 E^{-4}$ & $3.16 E^{-5}$ & $9.95 E^{-4}$ & $3.03 E^{-5}$ \\
\hline 1 & $\frac{1}{2}$ & $6.23 E^{-4}$ & $8.60 E^{-5}$ & $6.09 E^{-4}$ & $8.54 E^{-5}$ \\
\hline$\frac{1}{2}$ & $\frac{1}{4}$ & $3.13 E^{-4}$ & $1.02 E^{-4}$ & $2.74 E^{-4}$ & $1.01 E^{-4}$ \\
\hline$\frac{1}{4}$ & $\frac{1}{8}$ & $\mathrm{NaN}$ & $\mathrm{NaN}$ & $2.49 E^{-4}$ & $1.05 E^{-4}$ \\
\hline
\end{tabular}

Table 3. Depending on the used FEM error values are shown.

The instability of the Implicit Euler is shown in the last row of table 3 . In ge neral the error results are smaller compared to the results of the finite diffe rence method in one dimension. The finite element me thod a pproximates the conve ction-diffusion equation better than the finite difference method. 


\subsection{Analytical vs. Stochastic Results}

The acc uracy of the ra ndom $w$ alk a pproaches is discussed in the following paragraph.

One-dimensional. In the plot below in Figure 1 the red curve i s the analyti cal solution and the blue line sketches the numerical approximation using the Implicit Euler algorithm.

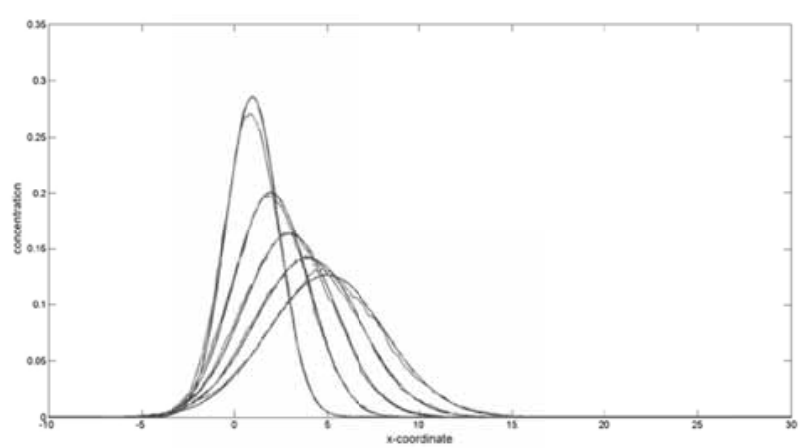

Figure 4. Results of stochastic based random walk are shown.

The gra phic i n Figure 4 sho $\mathrm{w}$ th e Gaussian $\mathrm{r}$ andom walk a pproach coloured in red and the analytical solution in blue. In the num erical comparisons the si mulation time is $t_{\text {end }}=500 \mathrm{~s}$. Due to long execution times for the particle movement this pa rameter is reduced to $t_{\text {end }}=250 \mathrm{~s}$. The diffusion coefficient is usually set to $D=0.02$ but $\mathrm{m}$ odifies if the $\mathrm{i}$ ntuitive ap proach i $\mathrm{s}$ used.

\begin{tabular}{|c|c|c|c|}
\hline$\Delta t$ & $\Delta x$ & $\begin{array}{l}\text { Gaussian } \\
\|\cdot\|_{\infty}\end{array}$ & $\begin{array}{c}\text { Random Walk } \\
\|\cdot\|_{1}\end{array}$ \\
\hline 1 & $\frac{1}{5}$ & 0.012 & $8.948 E^{-7}$ \\
\hline 1 & $\frac{1}{10}$ & 0.008 & $9.707 E^{-7}$ \\
\hline$\frac{1}{2}$ & $\frac{1}{5}$ & 0.007 & $8.948 E^{-7}$ \\
\hline$\frac{1}{2}$ & $\frac{1}{10}$ & 0.010 & $9.707 E^{-7}$ \\
\hline
\end{tabular}

Table 4. Comparison of random walk analytical solution.

The Table 4 shows all the error results of the para meter study comparing the a nalytical solution and the random walk. The diffusion co efficient for $t$ he Ga ussian-based algorithm is set to $D=0.02$. Regarding simulation of the convec tion-diffusion equation, the im plementation of the Gaussian-based random walk fits better tha $n$ the intuitive approach. The number of particles is 6000 .
Two-dimensional. In order to compare the analytical solution to a random walk ap proach the results have to be adapted. In the random wall the output describes the smoothed amount of pa rticles in every cel 1 . Due to the initial Dirac-fun ction the int egral at the beginning has value one. The area of the random walk domain is discretizised. Th erefore the output has to b e di vided n ot only by the nu mber of partic les but also $b$ y the area of the cells used for the flattening. Table 5 s hows the approximation results. The parameter $r$ describes the used radius for the flattening. If the spatial ste $\mathrm{p}$ size is decreasing a greater radius $r$ can be used. If $r$ is chosen too big compared to $\Delta x$ the result loses the shape of a b ell curve. Compared to the results of the num erical simulation the rand om walk appro ach lead s to greater e rror values. The number of particles is 4000 .

\begin{tabular}{cccccc}
\hline$\Delta t$ & $\Delta x$ & $r$ & $N$ & $\|\cdot\|_{\infty}$ & $\left\|_{1}\right\|_{1}$ \\
\hline 1 & 1 & 3 & 4000 & $3.395 E^{-3}$ & $6.349 E^{-4}$ \\
\hline 1 & $\frac{1}{2}$ & 8 & 4000 & $5.033 E^{-3}$ & $3.737 E^{-5}$ \\
\hline$\frac{1}{2}$ & $\frac{1}{4}$ & 15 & 4000 & $4.526 E^{-3}$ & $1.005 E^{-4}$ \\
\hline$\frac{1}{2}$ & $\frac{1}{8}$ & 20 & 4000 & $2.801 E^{-3}$ & $2.206 E^{-3}$ \\
\hline 1 & $\frac{1}{4}$ & 20 & 8000 & $6.764 E^{-3}$ & $1.826 E^{-4}$ \\
\hline
\end{tabular}

Table 5. Comparison of random walk analytical solution.

\section{Conclusion}

In general the finite elem ent method app roximates the convection-diffusion e quation the best. Of co urse the very best solution is the analytical one. In spite of it all random walk approaches are quite good approximations of the convection-diffusion equation.

\section{References}

[1] Schulten K, Kosztin I. Lectures in Theoretical Biophysics, University of Illinois at Urbana, USA; 2000.

[2] Zoppou C,Knight JH. Anal ytical solution of a spa tially variable coefficient advection diffusion equation in up to three dim ensions, Applied Mathematical Modelling; 1999. p 667-685.

[3] Segal A. Fin ite element methods for the incompressible Navier-Stokes equations, Delft University of Technology, Netherlands; 2012.

[4] Salamon P, Fernandez-Garcia D, Gomez-Hernandez JJ A review and numerical assessment of the random walk particle tr acking m ethod, Journal of Contaminant Hydrology; 2006. p 277-305. 